\title{
Short Sleep Duration Among Infants, Children, and Adolescents Aged 4 Months-17 Years — United States, 2016-2018
}

\author{
Anne G. Wheaton, $\mathrm{PhD}^{1}$; Angelika H. Claussen, $\mathrm{PhD}^{2}$
}

Infants, children, and adolescents who do not get sufficient sleep are at increased risk for injuries, obesity, type 2 diabetes, poor mental health, attention and behavior problems, and poor cognitive development (1). The American Academy of Sleep Medicine (AASM) provides age-specific sleep duration recommendations to promote optimal health (1). CDC analyzed data from the 2016-2018 National Survey of Children's Health (NSCH) to assess the prevalence of short sleep duration among persons in the United States aged 4 months-17 years. Overall, on the basis of parent report, $34.9 \%$ of persons aged 4 months-17 years slept less than recommended for their age. The prevalence of short sleep duration was higher in southeastern states and among racial and ethnic minority groups, persons with low socioeconomic status, and those with special health care needs. The prevalence of short sleep duration ranged from $31.2 \%$ among adolescents aged $13-17$ years to $40.3 \%$ among infants aged 4-11 months. Persons aged 4 months-17 years with a regular bedtime were more likely to get enough sleep. Public health practitioners, educators, and clinicians might advise parents on the importance of meeting recommended sleep duration and implementing a consistent bedtime for healthy development.

$\mathrm{NSCH}$ is a population-based, nationally representative online and paper survey of parents or primary caregivers (parents) of noninstitutionalized U.S. persons aged $\leq 17$ years. The survey is conducted by the U.S. Census Bureau under the direction of the Health Resources and Services Administration's Maternal and Child Health Bureau.* NSCH asks parents about the physical and emotional health of one person aged $\leq 17$ years selected at random from the household, as well as

\section{INSIDE}

1322 Increasing Access to HIV Testing Through Directto-Consumer HIV Self-Test Distribution - United States, March 31, 2020-March 30, 2021

1326 Nonvoluntary or Forced Sex Among Women, by Sexual Identity, Attraction, and Behavior National Survey of Family Growth, United States, 2011-2017

1332 Decreased Incidence of Infections Caused by Pathogens Transmitted Commonly Through Food During the COVID-19 Pandemic - Foodborne Diseases Active Surveillance Network, 10 U.S. Sites, 2017-2020

1337 Comparative Effectiveness of Moderna, PfizerBioNTech, and Janssen (Johnson \& Johnson) Vaccines in Preventing COVID-19 Hospitalizations Among Adults Without Immunocompromising Conditions - United States, March-August 2021

1344 Use of Pfizer-BioNTech COVID-19 Vaccine in Persons Aged $\geq 16$ Years: Recommendations of the Advisory Committee on Immunization Practices United States, September 2021

1349 Outbreak of SARS-CoV-2 B.1.617.2 (Delta) Variant Infections Among Incarcerated Persons in a Federal Prison — Texas, July-August 2021

1356 QuickStats

Continuing Education examination available at https://www.cdc.gov/mmwr/mmwr_continuingEducation.html

\footnotetext{
*https://mchb.hrsa.gov/
}

U.S. Department of Health and Human Services

Centers for Disease Control and Prevention 
health care access and family characteristics. The weighted overall response rates were $40.7 \%$ in $2016,37.4 \%$ in 2017 , and $43.1 \%$ in $2018 .^{\dagger}$ Sleep duration questions were "During the past week, how many hours of sleep did this child get on an average day (count both nighttime sleep and naps)?" $\$$ for infants and children aged $0-5$ years, and for children and adolescents aged 6-17 years, "During the past week, how many hours of sleep did this child get on an average weeknight?" On the basis of AASM recommendations (1), short sleep duration was defined as $<12$ hours for children aged 4-11 months, $<11$ hours for children aged $1-2$ years, $<10$ hours for children aged 3-5 years, $<9$ hours for children aged 6-12 years, and $<8$ hours for adolescents aged 13-17 years. The bedtime question for all ages was "How often does this child go to bed at about the same time on weeknights?"** Regular bedtime was defined as a response of "always." The study included 99,842

\footnotetext{
$\dagger$ https://www.census.gov/content/dam/Census/programs-surveys/nsch/techdocumentation/methodology/2016-NSCH-Methodology-Report.pdf; https://www.census.gov/content/dam/Census/programs-surveys/nsch/techdocumentation/methodology/2017-NSCH-Methodology-Report.pdf; https://www2.census.gov/programs-surveys/nsch/technical-documentation/ methodology/2018-NSCH-Methodology-Report.pdf

$\$$ Response options were "less than 7 hours," "7 hours," "8 hours," "9 hours," "10 hours," "11 hours," and " 12 or more hours."

9 The question changed slightly in 2018 , when parents were asked, "During the past week, how many hours of sleep did this child get on most weeknights?" Response options for 2016-2018 were "less than 6 hours," "6 hours," "7 hours," "8 hours," "9 hours," "10 hours," and "11 or more hours."

** Response options were "always," "usually," "sometimes," "rarely," and "never."
}

persons aged 4 months -17 years ${ }^{\dagger \dagger}$ with responses to the sleep duration question $(48,748$ in $2016,21,124$ in 2017 , and 29,970 in 2018).

Prevalence and 95\% confidence interval (CI) of short sleep duration and regular bedtime were calculated for persons aged 4 months -17 years overall, by age group, by state, and by selected characteristics of the child and parent. Pairwise differences by sex, race/ethnicity, special health care needs status, overall health status, and regular bedtime were determined using t-tests. Tests for linear trend were conducted for family income level ${ }^{\S \S}$ and parent education level. P-values $<0.05$ were considered statistically significant. Analyses accounted for weighting 99 of the data and for the complex sampling design. SAS-callable SUDAAN (version 11.0.3; RTI International) was used to conduct all analyses. This activity was reviewed by $\mathrm{CDC}$ and was conducted consistent with applicable federal law and CDC policy.***

Overall, $34.9 \%$ of persons aged 4 months- 17 years slept less than recommended for their age (Table 1). The prevalence of short sleep duration ranged from $31.2 \%$ among adolescents aged $13-17$ years to $40.3 \%$ among infants aged $4-11$ months.

\footnotetext{
†† AASM did not include recommendations for persons aged $<4$ months; therefore, short sleep duration was not assessed for this age group.

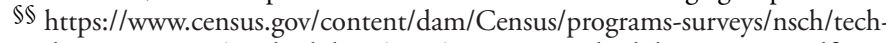
documentation/methodology/2016-NSCH-Methodology-Report.pdf

99 Weighted estimates were used to generalize to state and national resident populations.

*** 45 C.F.R. part 46.102(1)(2), 21 C.F.R. part 56; 42 U.S.C. Sect. 241(d); 5 U.S.C. Sect. 552a; 44 U.S.C. Sect. 3501 et seq.
}

The MMWR series of publications is published by the Center for Surveillance, Epidemiology, and Laboratory Services, Centers for Disease Control and Prevention (CDC), U.S. Department of Health and Human Services, Atlanta, GA 30329-4027.

Suggested citation: [Author names; first three, then et al., if more than six.] [Report title]. MMWR Morb Mortal Wkly Rep 2021;70:[inclusive page numbers].

\section{Centers for Disease Control and Prevention \\ Rochelle P. Walensky, MD, MPH, Director \\ Debra Houry, MD, MPH, Acting Principal Deputy Director \\ Daniel B. Jernigan, MD, MPH, Deputy Director for Public Health Science and Surveillance \\ Rebecca Bunnell, PhD, MEd, Director, Office of Science \\ Jennifer Layden, MD, PhD, Deputy Director, Office of Science \\ Michael F. Iademarco, MD, MPH, Director, Center for Surveillance, Epidemiology, and Laboratory Services \\ MMWR Editorial and Production Staff (Weekly)}

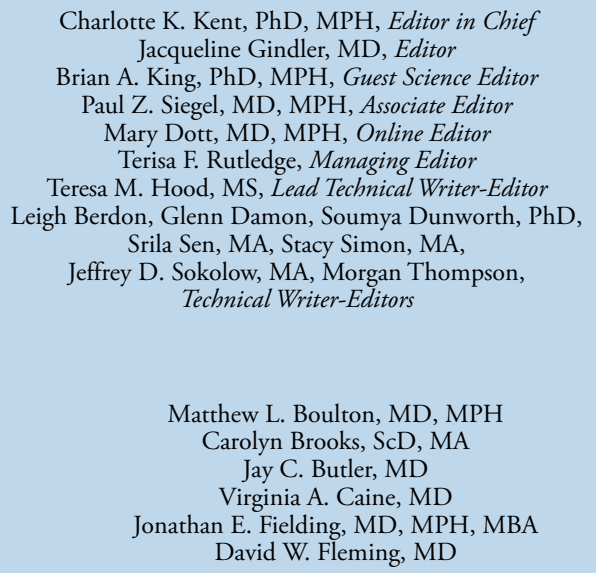

Charlotte K. Kent, PhD, MPH, Editor in Chief Jacqueline Gindler, MD, Editor

Brian A. King, PhD, MPH, Guest Science Editor

Paul Z. Siegel, MD, MPH, Associate Editor

Mary Dott, MD, MPH, Online Editor

Terisa F. Rutledge, Managing Editor

Teresa M. Hood, MS, Lead Technical Writer-Editor

Leigh Berdon, Glenn Damon, Soumya Dunworth, PhD, Srila Sen, MA, Stacy Simon, MA,

Jeffrey D. Sokolow, MA, Morgan Thompson, Technical Writer-Editors

Matthew L. Boulton, MD, MPH Carolyn Brooks, ScD, MA

Jay C. Butler, MD Virginia A. Caine, MD

Jonathan E. Fielding, MD, MPH, MBA David W. Fleming, MD

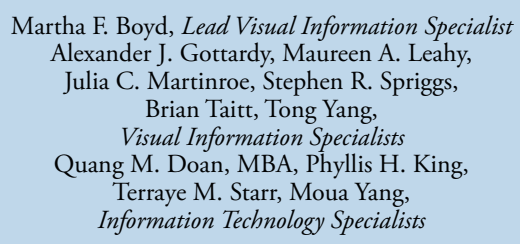

Ian Branam, MA,

Acting Lead Health Communication Specialist Shelton Bartley, MPH, Lowery Johnson, Amanda Ray, Jacqueline N. Sanchez, MS, Health Communication Specialists Will Yang, MA, Visual Information Specialist 
TABLE 1. Prevalence of short sleep duration* and regular bedtime ${ }^{\dagger}$ among persons aged 4 months-17 years, by age group - National Survey of Children's Health, United States, 2016-2018

\begin{tabular}{|c|c|c|c|c|c|c|}
\hline \multirow[b]{2}{*}{ Characteristic } & \multicolumn{6}{|c|}{$\%(95 \% \mathrm{Cl})^{\S}$} \\
\hline & $\begin{array}{c}\text { All persons aged } \\
4 \text { mos-17 yrs } \\
(n=99,842)\end{array}$ & $\begin{array}{c}\text { Infants aged } \\
4-11 \text { mos } \\
(n=2,499)\end{array}$ & $\begin{array}{c}\text { Children aged } \\
\begin{array}{c}1-2 \text { yrs } \\
(n=10,147)\end{array}\end{array}$ & $\begin{array}{l}\text { Children aged } \\
\text { 3-5 yrs } \\
(n=15,290)\end{array}$ & $\begin{array}{l}\text { Children aged } \\
\text { 6-12 yrs } \\
(n=36,179)\end{array}$ & $\begin{array}{c}\text { Adolescents aged } \\
13-17 \text { yrs } \\
(n=35,727)\end{array}$ \\
\hline Short sleep duration & $34.9(34.2-35.6)$ & $40.3(35.9-44.7)$ & $33.3(31.2-35.4)$ & $34.8(33.1-36.7)$ & $37.4(36.3-38.6)$ & $31.2(30.1-32.4)$ \\
\hline Regular bedtime & $33.9(33.2-34.6)$ & 43.5 (39.1-47.9) & $40.9(38.7-43.0)$ & $37.3(35.5-39.1)$ & $37.0(35.9-38.2)$ & $23.8(22.7-24.9)$ \\
\hline
\end{tabular}

Abbreviation: $\mathrm{Cl}=$ confidence interval.

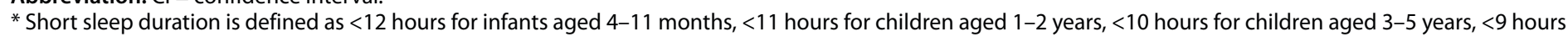
for children aged 6-12 years, and $<8$ hours for adolescents aged 13-17 years.

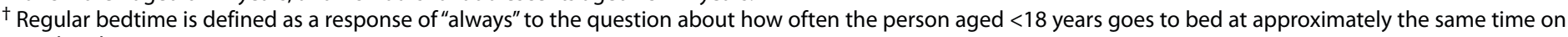
weeknights.

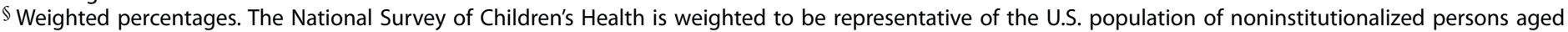
$\leq 17$ years. https://www2.census.gov/programs-surveys/nsch/technical-documentation/methodology/NSCH-Guide-to-Multi-Year-Estimates.pdf

The percentage of persons aged 4 months-17 years with a regular bedtime was $33.9 \%$ overall and ranged from $23.8 \%$ among adolescents to $43.5 \%$ among infants. Among children aged 4 months-17 years, the prevalence of short sleep duration was lowest among non-Hispanic White children $(28.8 \%)$ and higher among non-Hispanic Black children (50.8\%) than among Hispanic children $(39.1 \%)$ or non-Hispanic children of any other race (other race) (34.6\%) (Table 2). The prevalence decreased with increasing family income level and parent educational attainment. The prevalence of short sleep duration was higher among persons aged 4 months -17 years with special health care needs than among those without special health care needs (38.3\% versus $34.1 \%$ ); and among those with good, fair, or poor health than among those with excellent or very good health ( $46.8 \%$ versus $33.5 \%)$. Overall, the prevalence of short sleep duration was lower among persons aged 4 months -17 years with a regular bedtime than among those without a regular bedtime (27.5\% versus $38.6 \%)$. Similar patterns were observed within each age group.

State-level prevalence of short sleep duration overall ranged from $25.3 \%$ in Maine to $48.9 \%$ in Mississippi (Table 2). States with the highest prevalence of short sleep duration were concentrated in the Southeast (Figure).

\section{Discussion}

During 2016-2018, approximately one third of persons aged 4 months-17 years (34.9\%) got less sleep than is recommended for their age (1). Younger persons within this age group were particularly at risk for short sleep duration; the prevalence of short sleep duration decreased with age from infancy $(40.3 \%)$ to adolescence $(31.2 \%)$. Previous prevalence estimates of short sleep duration among adolescents were significantly higher: based on self-report from the 2015 Youth Risk Behavior Surveys, ${ }^{\dagger \dagger \dagger}$ nearly three quarters of high school

\footnotetext{
$\overline{\dagger \dagger}$ https://www.cdc.gov/healthyyouth/data/yrbs/index.htm
}

students nationally and approximately one half of middle school students in nine states reported getting less sleep than recommended for their age (2). This difference might be explained by NSCH's reliance on parent report rather than self-report with Youth Risk Behavior Surveys. Parents might overestimate the amount of sleep their older children or adolescents receive (3). However, one study that compared total sleep time based on parent report and child or adolescent report with that measured by a sleep study (i.e., polysomnography $\$ \$ S$ ) found that children and adolescents overestimated total sleep time to a lesser extent than did their parents, but the difference between child or adolescent and parent reports was small (4). Agreement between parent and child or adolescent report was similar for children aged 9-12 years and adolescents aged 13-17 years (4).

Before 2016, the NSCH did not ask parents about hours of sleep, but rather asked, "During the past week, on how many nights did [child] get enough sleep for a child his/her age?" Patterns in the prevalence of inadequate sleep (defined as not enough sleep $\geq 1$ night during the past week) contrast with the current report; specifically, the prevalence of inadequate sleep was highest among adolescents aged 14-17 years and lowest among children aged 6-9 years (5). Trends based on parent education and household income also differed (5).

In the current study, short sleep duration was elevated among racial and ethnic minority groups, especially among Black persons aged 4 months -17 years, among whom approximately one half had short sleep duration. Short sleep duration was also more prevalent among families with lower income or lower parental educational attainment. In previous research, sleep disparity was associated with various social determinants of health (e.g., poverty, food insecurity, and perceived racism), which can increase chronic and acute stress and result in environmental and psychological factors that negatively affect sleep

$\overline{\$ \$ \$ \text { https://www.nhlbi.nih.gov/health-topics/sleep-studies }}$ 
TABLE 2. Prevalence of short sleep duration* among persons aged 4 months-17 years, by age group and selected characteristics — National Survey of Children's Health, United States, 2016-2018

\begin{tabular}{|c|c|c|c|c|c|c|}
\hline \multirow[b]{2}{*}{ Characteristic } & \multicolumn{6}{|c|}{$\%(95 \% \mathrm{Cl})^{\dagger}$} \\
\hline & $\begin{array}{l}\text { All persons aged } \\
4 \text { mos- }-17 \text { yrs }\end{array}$ & $\begin{array}{l}\text { Infants aged } \\
4-11 \text { mos }\end{array}$ & $\begin{array}{l}\text { Children aged } \\
1-2 \text { yrs }\end{array}$ & $\begin{array}{l}\text { Children aged } \\
3-5 \text { yrs }\end{array}$ & $\begin{array}{l}\text { Children aged } \\
6-12 \text { yrs }\end{array}$ & $\begin{array}{c}\text { Adolescents aged } \\
13-17 \text { yrs }\end{array}$ \\
\hline \multicolumn{7}{|l|}{ Sex } \\
\hline Male & $35.0(34.0-36.0)$ & $40.6(34.4-47.0)$ & $32.8(29.9-35.7)$ & $35.2(32.8-37.7)$ & $38.4(36.8-40.0)$ & $30.4(28.9-32.1)$ \\
\hline Female & $34.7(33.7-35.7)$ & $39.9(34.0-46.1)$ & $33.8(30.8-36.8)$ & 34.5 (31.9-37.1) & $36.4(34.8-38.1)$ & $32.1(30.3-33.8)$ \\
\hline \multicolumn{7}{|l|}{ Race/Ethnicity } \\
\hline Hispanic & $39.1(37.1-41.1)$ & $41.5(29.9-54.1)^{\S}$ & $40.5(34.6-46.7)$ & $42.4(37.3-47.7)$ & $43.3(40.1-46.6)$ & $30.3(27.1-33.7)$ \\
\hline White, non-Hispanic & $28.8(28.1-29.5)$ & $32.9(28.2-38.0)$ & $26.1(24.2-28.2)$ & $27.9(26.2-29.7)$ & $30.1(29.0-31.1)$ & $28.1(27.1-29.2)$ \\
\hline Black, non-Hispanic & $50.8(48.6-53.0)$ & $64.2(51.1-75.5)^{\S}$ & $50.2(42.7-57.7)$ & $51.7(46.1-57.4)$ & $56.4(53.0-59.8)$ & $42.2(38.5-46.0)$ \\
\hline Other, ${ }^{9}$ non-Hispanic & $34.6(32.9-36.4)$ & $46.8(36.8-57.2)^{\S}$ & $35.6(29.7-41.9)$ & $32.3(28.7-36.1)$ & $34.8(32.1-37.6)$ & $33.4(30.5-36.5)$ \\
\hline \multicolumn{7}{|l|}{ Family income level ${ }^{* *}$} \\
\hline $\mathrm{FPL}<100 \%$ & $45.6(43.6-47.7)$ & $54.2(41.5-66.4)^{\S}$ & $45.4(39.5-51.5)$ & $49.2(44.1-54.3)$ & $51.6(48.4-54.8)$ & $33.4(30.1-36.9)$ \\
\hline $100 \% \leq \mathrm{FPL}<200 \%$ & $39.2(37.3-41.2)$ & $49.9(39.6-60.2)^{\S}$ & $39.1(33.6-44.8)$ & $41.4(36.4-46.5)$ & $41.1(38.1-44.1)$ & $34.0(30.9-37.2)$ \\
\hline $200 \% \leq F P L<400 \%$ & $33.2(31.9-34.6)$ & $33.3(25.3-42.4)$ & $32.7(28.3-37.3)$ & $32.7(29.4-36.2)$ & $35.8(33.7-37.9)$ & $30.4(28.3-32.5)$ \\
\hline $\mathrm{FPL} \geq 400 \%$ & $26.0(25.0-27.0)$ & $29.2(23.7-35.5)$ & $21.9(18.9-25.4)$ & $22.5(20.1-25.0)$ & $26.3(24.8-27.7)$ & $28.7(27.1-30.5)$ \\
\hline \multicolumn{7}{|l|}{ Parent education } \\
\hline Less than high school & $46.2(42.4-50.0)$ & $60.3(34.7-81.2)^{\S}$ & $39.7(27.8-53.0)^{\S}$ & $54.0(43.1-64.5)^{\S}$ & $53.7(47.7-59.5)$ & $34.1(28.6-40.1)$ \\
\hline High school & $43.3(41.4-45.2)$ & $54.1(43.8-64.1)^{\S}$ & $49.0(42.7-55.4)$ & $47.4(42.2-52.7)$ & $46.3(43.3-49.3)$ & $34.2(31.2-37.3)$ \\
\hline Some college & $39.3(37.8-40.7)$ & $49.2(40.3-58.2)$ & $39.2(34.7-44.0)$ & $40.6(36.8-44.4)$ & $43.0(40.7-45.3)$ & $32.2(30.0-34.5)$ \\
\hline College graduate & $27.2(26.5-28.0)$ & $28.6(24.3-33.3)$ & $24.9(22.6-27.3)$ & $25.0(23.2-26.8)$ & $27.8(26.6-29.0)$ & $28.7(27.4-30.1)$ \\
\hline \multicolumn{7}{|c|}{ Special health care needs ${ }^{\dagger \dagger}$} \\
\hline Yes & $38.3(36.8-39.7)$ & $30.5(19.5-44.2)^{\S}$ & $30.9(25.3-37.1)$ & $38.3(34.3-42.6)$ & $42.8(40.7-45.1)$ & $33.7(31.4-36.1)$ \\
\hline No & $34.1(33.3-34.9)$ & $40.7(36.2-45.3)$ & $33.5(31.3-35.7)$ & $34.3(32.3-36.3)$ & $35.9(34.6-37.3)$ & $30.4(29.1-31.8)$ \\
\hline \multicolumn{7}{|l|}{ Health status } \\
\hline Excellent or very good & $33.5(32.8-34.2)$ & $39.4(35.0-44.0)$ & $32.4(30.4-34.5)$ & 33.8 (31.9-35.6) & $35.3(34.2-36.5)$ & $30.3(29.1-31.5)$ \\
\hline Good, fair, or poor & $46.8(44.1-49.5)$ & $59.8(40.2-76.7)^{\S}$ & $45.4(35.1-56.1)^{\S}$ & $45.6(38.1-53.3)$ & $54.6(50.5-58.7)$ & $38.0(33.8-42.4)$ \\
\hline \multicolumn{7}{|l|}{ Regular bedtime $\mathrm{s}^{\S \S}$} \\
\hline Yes & $27.5(26.3-28.9)$ & $34.6(27.6-42.3)$ & $28.5(25.5-31.7)$ & $29.1(25.8-32.6)$ & $30.5(28.6-32.6)$ & $17.4(15.5-19.6)$ \\
\hline No & $38.6(37.8-39.4)$ & $44.8(39.5-50.2)$ & $36.6(33.8-39.4)$ & $38.1(36.0-40.2)$ & $41.4(40.0-42.8)$ & $35.6(34.2-37.0)$ \\
\hline \multicolumn{7}{|l|}{ State } \\
\hline Alabama & $42.5(39.3-45.8)$ & $46.7(28.3-66.2)^{\S}$ & $41.7(32.0-52.0)$ & $39.2(32.1-46.8)$ & $49.6(44.3-54.9)$ & 34.4 (29.3-39.9) \\
\hline Alaska & $27.7(24.7-30.8)$ & $32.1(19.6-47.8)^{\S}$ & $28.3(20.8-37.3)$ & $26.6(20.1-34.2)$ & $31.9(26.9-37.4)$ & $20.5(16.2-25.8)$ \\
\hline Arizona & $35.6(32.3-38.9)$ & $27.1(13.8-46.2)^{\S}$ & $36.7(27.1-47.4)^{\S}$ & $35.9(28.1-44.5)$ & $38.1(32.9-43.6)$ & $32.2(26.8-38.0)$ \\
\hline Arkansas & $40.8(37.4-44.2)$ & $47.6(31.5-64.1)^{\S}$ & $35.7(26.3-46.4)^{\S}$ & $45.9(37.7-54.4)$ & $44.2(38.8-49.7)$ & $34.0(28.2-40.4)$ \\
\hline California & $34.2(30.9-37.6)$ & $44.9(24.2-67.6)^{\S}$ & $30.3(22.0-40.1)$ & $33.8(26.1-42.4)$ & $38.2(32.9-43.8)$ & $28.5(23.4-34.2)$ \\
\hline Colorado & $26.8(24.1-29.7)$ & $27.1(15.7-42.6)^{\S}$ & $25.8(17.6-36.1)$ & $28.2(22.0-35.3)$ & $26.9(22.6-31.6)$ & $26.3(21.7-31.6)$ \\
\hline Connecticut & $32.8(29.9-36.0)$ & $46.5(27.0-67.0)^{\S}$ & $27.8(19.0-38.6)$ & 36.7 (28.5-45.7) & $31.7(27.1-36.7)$ & $32.8(28.2-37.8)$ \\
\hline Delaware & $39.1(35.9-42.5)$ & $46.2(29.0-64.3)^{\S}$ & $35.9(26.4-46.6)^{\S}$ & $42.4(34.0-51.3)$ & $42.1(37.0-47.3)$ & $33.5(28.4-39.0)$ \\
\hline District of Columbia & $36.5(32.8-40.3)$ & $31.2(17.9-48.6)^{\S}$ & 36.7 (27.7-46.7) & 25.9 (19.4-33.7) & $44.5(38.0-51.1)$ & 34.7 (27.5-42.6) \\
\hline Florida & $38.6(35.3-41.9)$ & $50.0(29.0-71.0)^{\S}$ & $36.0(27.5-45.6)$ & $43.3(34.6-52.5)$ & $38.4(33.4-43.6)$ & $36.2(30.5-42.2)$ \\
\hline Georgia & $40.3(37.0-43.6)$ & $38.9(22.2-58.7)^{\S}$ & $33.5(24.2-44.3)^{\S}$ & $39.4(31.4-48.1)$ & $47.1(41.8-52.5)$ & $32.9(28.0-38.2)$ \\
\hline Hawaii & $38.4(35.5-41.3)$ & $41.6(26.2-58.9)^{\S}$ & $32.2(23.9-41.7)$ & $32.2(25.6-39.6)$ & $43.0(38.3-47.9)$ & $38.4(33.6-43.5)$ \\
\hline Idaho & $26.9(24.4-29.6)$ & $22.0(12.5-35.6)^{\S}$ & $25.0(18.5-32.8)$ & $28.6(21.9-36.4)$ & $28.4(24.3-32.9)$ & $25.1(21.2-29.5)$ \\
\hline Illinois & $30.1(27.2-33.1)$ & $53.4(36.1-69.9)^{\S}$ & $23.6(16.5-32.6)$ & $27.9(20.7-36.4)$ & $32.2(27.5-37.2)$ & $28.4(23.7-33.5)$ \\
\hline Indiana & $38.6(35.5-41.7)$ & $36.7(20.8-56.0)^{\S}$ & $45.8(36.6-55.3)$ & $35.4(28.3-43.2)$ & $40.4(35.3-45.6)$ & $36.0(31.0-41.3)$ \\
\hline lowa & $29.0(26.3-32.0)$ & $28.7(12.9-52.4)^{\S}$ & $20.5(13.3-30.3)$ & $28.9(22.6-36.2)$ & $34.3(29.6-39.4)$ & $25.0(21.3-29.2)$ \\
\hline Kansas & $30.3(27.3-33.4)$ & $39.9(21.3-62.0)^{\S}$ & $27.0(18.0-38.5)^{\S}$ & $31.0(24.2-38.8)$ & $32.5(27.9-37.5)$ & $26.7(22.2-31.8)$ \\
\hline Kentucky & $35.8(32.8-39.0)$ & $28.9(12.8-53.0)^{\S}$ & $31.0(23.2-40.2)$ & $28.8(22.3-36.3)$ & $46.0(40.6-51.5)$ & $29.4(25.1-34.3)$ \\
\hline Louisiana & $46.6(43.2-49.9)$ & $34.0(19.7-51.9)^{\S}$ & $44.9(34.8-55.6)^{\S}$ & $47.0(39.3-54.9)$ & $53.1(47.7-58.5)$ & $39.0(33.5-44.9)$ \\
\hline Maine & $25.3(22.7-28.1)$ & $29.0(14.1-50.3)^{\S}$ & $24.9(17.2-34.7)$ & $20.4(14.9-27.4)$ & $25.6(21.3-30.4)$ & $27.8(23.6-32.3)$ \\
\hline Maryland & $37.4(34.3-40.7)$ & $16.7(6.9-35.0)^{\S}$ & $36.7(27.3-47.2)$ & $43.5(35.4-51.8)$ & $41.8(36.8-47.1)$ & $30.5(25.7-35.8)$ \\
\hline Massachusetts & $30.0(27.1-33.1)$ & $35.9(21.1-54.0)^{\S}$ & $25.7(17.3-36.3)$ & $30.7(23.4-39.1)$ & $30.9(26.1-36.2)$ & $29.5(24.9-34.5)$ \\
\hline Michigan & $32.4(29.5-35.6)$ & $40.1(22.0-61.3)^{\S}$ & 29.7 (21.2-39.9) & $34.5(27.0-42.9)$ & $33.9(29.1-39.0)$ & $29.6(25.1-34.6)$ \\
\hline Minnesota & $25.4(22.6-28.4)$ & $37.9(22.9-55.6)^{\S}$ & $27.4(17.8-39.7)^{\S}$ & $27.3(20.9-34.8)$ & $26.2(21.9-31.1)$ & $20.6(16.3-25.6)$ \\
\hline Mississippi & $48.9(45.5-52.2)$ & $52.6(30.0-74.2)^{\S}$ & 45.1 (35.9-54.7) & $44.5(36.6-52.6)$ & $60.7(55.5-65.7)$ & $36.2(30.8-42.0)$ \\
\hline Missouri & 35.8 (32.7-38.9) & $24.0(12.7-40.8)^{\S}$ & $35.9(26.8-46.1)$ & $37.0(29.8-44.8)$ & $37.6(32.5-42.9)$ & 33.8 (29.1-38.8) \\
\hline Montana & $27.5(24.7-30.5)$ & $35.5(20.6-53.9)^{\S}$ & $30.4(21.7-40.7)$ & $29.1(22.4-36.9)$ & $28.0(23.4-33.1)$ & 23.5 (19.6-27.9) \\
\hline Nebraska & $30.8(27.7-34.0)$ & $49.8(31.8-67.9)^{\S}$ & $29.1(21.3-38.3)$ & $26.0(19.8-33.2)$ & $31.0(26.3-36.2)$ & $31.2(25.6-37.4)$ \\
\hline Nevada & $32.4(29.2-35.6)$ & $33.1(19.0-51.2)^{\S}$ & $33.4(24.1-44.3)^{\S}$ & $28.9(22.1-36.8)$ & $35.9(30.8-41.3)$ & $29.1(23.7-35.1)$ \\
\hline New Hampshire & $27.0(24.5-29.7)$ & $28.9(15.9-46.7)^{\S}$ & $23.1(14.4-34.8)^{\S}$ & $23.4(17.7-30.3)$ & $26.4(22.5-30.6)$ & $30.8(26.7-35.4)$ \\
\hline
\end{tabular}

See table footnotes on the next page. 
TABLE 2. (Continued) Prevalence of short sleep duration* among persons aged 4 months-17 years, by age group and selected characteristics - National Survey of Children's Health, United States, 2016-2018

\begin{tabular}{|c|c|c|c|c|c|c|}
\hline \multirow[b]{2}{*}{ Characteristic } & \multicolumn{6}{|c|}{$\%(95 \% \mathrm{Cl})^{\dagger}$} \\
\hline & $\begin{array}{l}\text { All persons aged } \\
4 \mathrm{mos}-17 \mathrm{yrs}\end{array}$ & $\begin{array}{l}\text { Infants aged } \\
4-11 \text { mos }\end{array}$ & $\begin{array}{c}\text { Children aged } \\
1-2 \text { yrs }\end{array}$ & $\begin{array}{c}\text { Children aged } \\
3-5 \text { yrs }\end{array}$ & $\begin{array}{l}\text { Children aged } \\
6-12 \text { yrs }\end{array}$ & $\begin{array}{c}\text { Adolescents aged } \\
13-17 \mathrm{yrs}\end{array}$ \\
\hline New Jersey & $34.6(31.5-37.9)$ & $41.4(21.3-64.8)^{\S}$ & $34.8(24.8-46.3)^{\S}$ & $37.7(30.2-45.9)$ & $33.0(28.0-38.3)$ & $34.2(29.1-39.6)$ \\
\hline New Mexico & $33.8(30.4-37.2)$ & $42.5(23.9-63.5)^{\S}$ & $34.5(24.8-45.8)^{\S}$ & $35.9(28.1-44.5)$ & $35.7(30.3-41.5)$ & $28.6(23.2-34.6)$ \\
\hline New York & $36.2(32.9-39.6)$ & $39.5(22.0-60.2)^{\S}$ & $25.5(17.5-35.4)$ & $32.2(24.7-40.8)$ & $39.7(34.4-45.3)$ & $37.4(31.7-43.5)$ \\
\hline North Carolina & $36.8(33.5-40.3)$ & $48.6(31.2-66.2)^{\S}$ & $36.5(26.3-48.0)^{\S}$ & $38.9(31.2-47.3)$ & $37.7(32.4-43.4)$ & $33.0(27.6-38.9)$ \\
\hline North Dakota & $25.4(22.6-28.4)$ & $24.1(13.3-39.8)^{\S}$ & $33.4(24.4-43.8)$ & $24.1(17.7-31.8)$ & $24.6(20.2-29.6)$ & $23.2(19.3-27.7)$ \\
\hline Ohio & $34.8(31.8-38.0)$ & $44.3(24.8-65.7)^{\S}$ & $32.9(23.8-43.5)$ & $26.3(20.2-33.4)$ & $38.7(33.7-43.9)$ & $34.2(29.0-39.8)$ \\
\hline Oklahoma & $35.3(32.3-38.5)$ & $37.1(20.7-57.1)^{\S}$ & $42.3(32.5-52.6)^{\S}$ & $30.6(23.9-38.2)$ & $36.0(31.3-40.9)$ & $34.2(29.0-39.8)$ \\
\hline Oregon & $27.6(24.6-30.7)$ & $31.9(18.0-50.1)^{\S}$ & $33.5(23.9-44.7)^{\S}$ & $35.8(27.7-44.8)$ & $23.9(19.7-28.7)$ & $25.3(20.8-30.5)$ \\
\hline Pennsylvania & $32.8(29.9-35.9)$ & $40.9(24.3-59.8)^{\S}$ & $27.5(20.4-36.0)$ & $32.9(25.6-41.2)$ & $35.9(31.0-41.0)$ & $29.0(24.5-33.9)$ \\
\hline Rhode Island & $33.9(30.8-37.1)$ & $58.2(40.0-74.4)^{\S}$ & $25.5(18.4-34.2)$ & $30.2(22.9-38.6)$ & $35.5(30.6-40.7)$ & $33.7(28.3-39.5)$ \\
\hline South Carolina & $40.2(37.0-43.5)$ & $30.6(12.8-57.1)^{\S}$ & $45.6(36.2-55.4)$ & $41.8(33.5-50.5)$ & $41.7(36.6-47.0)$ & $35.5(30.2-41.1)$ \\
\hline South Dakota & $30.0(27.2-32.9)$ & $46.8(33.0-61.2)^{\S}$ & $29.1(22.3-37.0)$ & $31.3(24.4-39.1)$ & $28.9(24.4-33.9)$ & $27.7(23.1-32.8)$ \\
\hline Tennessee & $39.1(36.0-42.4)$ & $58.3(39.8-74.7)^{\S}$ & $41.5(32.4-51.1)$ & $39.7(32.4-47.6)$ & $42.5(37.4-47.7)$ & $30.2(25.5-35.4)$ \\
\hline Texas & $36.7(33.3-40.2)$ & $35.3(21.1-52.6)^{\S}$ & $41.8(32.0-52.4)^{\S}$ & $40.4(31.9-49.6)$ & $38.0(32.6-43.8)$ & $30.7(25.0-37.1)$ \\
\hline Utah & $29.3(26.5-32.3)$ & $25.9(14.0-42.8)^{\S}$ & $31.6(22.6-42.4)$ & $24.0(18.6-30.3)$ & $32.3(27.7-37.3)$ & $28.0(23.1-33.5)$ \\
\hline Vermont & $25.6(22.9-28.6)$ & $47.5(29.4-66.2)^{\S}$ & $26.5(19.0-35.5)$ & $26.6(19.8-34.7)$ & $25.6(21.3-30.5)$ & $21.8(17.9-26.2)$ \\
\hline Virginia & $32.7(29.7-35.8)$ & $45.1(26.0-65.7)^{\S}$ & $33.1(24.0-43.6)$ & $32.2(25.0-40.4)$ & $35.1(30.3-40.3)$ & $28.5(24.0-33.4)$ \\
\hline Washington & $30.5(27.5-33.7)$ & $43.4(26.6-61.9)^{\S}$ & $32.4(23.4-42.9)$ & $30.4(23.6-38.3)$ & $31.3(26.3-36.7)$ & $27.1(22.1-32.7)$ \\
\hline West Virginia & $42.9(39.7-46.3)$ & $45.2(30.0-61.2)^{\S}$ & $47.4(36.3-58.8)^{\S}$ & $46.2(38.3-54.3)$ & $42.9(37.9-48.1)$ & $38.8(33.4-44.5)$ \\
\hline Wisconsin & $28.9(26.2-31.9)$ & $46.3(28.0-65.8)^{\S}$ & $26.5(19.0-35.8)$ & $23.1(17.3-30.3)$ & $30.6(26.3-35.4)$ & $28.5(24.0-33.4)$ \\
\hline Wyoming & $31.9(28.9-35.0)$ & $32.3(18.0-50.8)^{\S}$ & $33.6(25.6-42.7)$ & $30.2(23.1-38.3)$ & $36.4(31.5-41.7)$ & $25.1(20.6-30.1)$ \\
\hline
\end{tabular}

Abbreviations: $\mathrm{Cl}=$ confidence interval; $\mathrm{FPL}=$ federal poverty level.

* Short sleep duration is defined as $<12$ hours for infants aged $4-11$ months, $<11$ hours for children aged $1-2$ years, $<10$ hours for children aged $3-5$ years, $<9$ hours for children aged 6-12 years, and $<8$ hours for adolescents aged $13-17$ years.

† Weighted percentages. Weighted estimates generalize to state and national resident populations. https://www2.census.gov/programs-surveys/nsch/technicaldocumentation/methodology/NSCH-Guide-to-Multi-Year-Estimates.pdf; https://www.census.gov/content/dam/Census/programs-surveys/nsch/techdocumentation/methodology/2016-NSCH-Methodology-Report.pdf; https://www.census.gov/content/dam/Census/programs-surveys/nsch/tech-documentation/ methodology/2017-NSCH-Methodology-Report.pdf; https://www2.census.gov/programs-surveys/nsch/technical-documentation/methodology/2018-NSCHMethodology-Report.pdf

$\S$ Estimate might be unreliable. The absolute $\mathrm{Cl}$ width is $>20 \%$ or the relative $\mathrm{Cl}$ width is $>120 \%$ ( 1.2 times the estimate). https://www.childhealthdata.org/docs/ default-source/drc/nsch_data-supression-and-display_revised_03-01-19.pdf

II Includes American Indian or Alaska Native, Native Hawaiian or Other Pacific Islander, Asian, other race, or multiracial.

** FPL is based on family income and family size and composition using the U.S. Census Bureau's poverty thresholds. Imputed income was used for persons aged 4 months-17 years without reported family income. https://www.census.gov/topics/income-poverty/poverty/guidance/poverty-measures.html

t+ Special health care needs status is based on responses to the Children with Special Health Care Needs standardized five-item screener that included 1) the need for or use of medications (other than vitamins) prescribed by a doctor; 2 ) the need for or use of medical care, mental health, or educational services beyond those of a similarly aged person (referred to as "average use"); 3) limitation in the ability to do things most persons of the same age can do; 4) the need for or use of specialized therapies such as physical, occupational, or speech therapy; and 5) the need for or receipt of treatment or counseling for an emotional, behavioral, or developmental problem. https://www.census.gov/content/dam/Census/programs-surveys/nsch/tech-documentation/methodology/2016-NSCH-MethodologyReport.pdf; https://www.census.gov/content/dam/Census/programs-surveys/nsch/tech-documentation/methodology/2017-NSCH-Methodology-Report.pdf; https://www2.census.gov/programs-surveys/nsch/technical-documentation/methodology/2018-NSCH-Methodology-Report.pdf

$\S \S$ Regular bedtime is defined as a response of "always" to the question about how often the person aged 4 months-17 years goes to bed at approximately the same time on weeknights.

duration and can compound long-term health risks (G). Some parents, particularly those affected by socioeconomic and racial disparities, might face additional challenges to ensuring their infants, children, and adolescents get sufficient, quality sleep. For example, parents who work multiple jobs or do shift work might have difficulty implementing a consistent bedtime ( $\sigma$ ). In addition, a family's housing situation could make achieving a quiet, comfortable sleep environment difficult because of noise, lack of sleeping space, or disruptive, unsafe, or violent neighborhoods (G).

The prevalence of short sleep duration was higher among persons aged 4 months-17 years whose current health was rated less positively and among those with special health care needs.
Multiple conditions, including attention deficit/hyperactivity syndrome or other neurodevelopmental disorders, have been associated with sleep problems as well as sleep behaviors that might be amenable to behavioral intervention ( 7$)$.

The prevalence of short sleep duration was highest in the Southeast, similar to geographic variation in adequate sleep observed for adults (8). This pattern might be partially explained by a higher prevalence of risks associated with poverty and racial and ethnic minority status in these states. 999 In a previous report of short sleep duration among high school students in 30 states, most of the states with a high prevalence of short sleep duration

$\overline{999}$ https://www.census.gov/library/visualizations/interactive.html 
FIGURE. Prevalence of short sleep duration* among persons aged 4 months -17 years, by state - National Survey of Children's Health, United States, 2016-2018

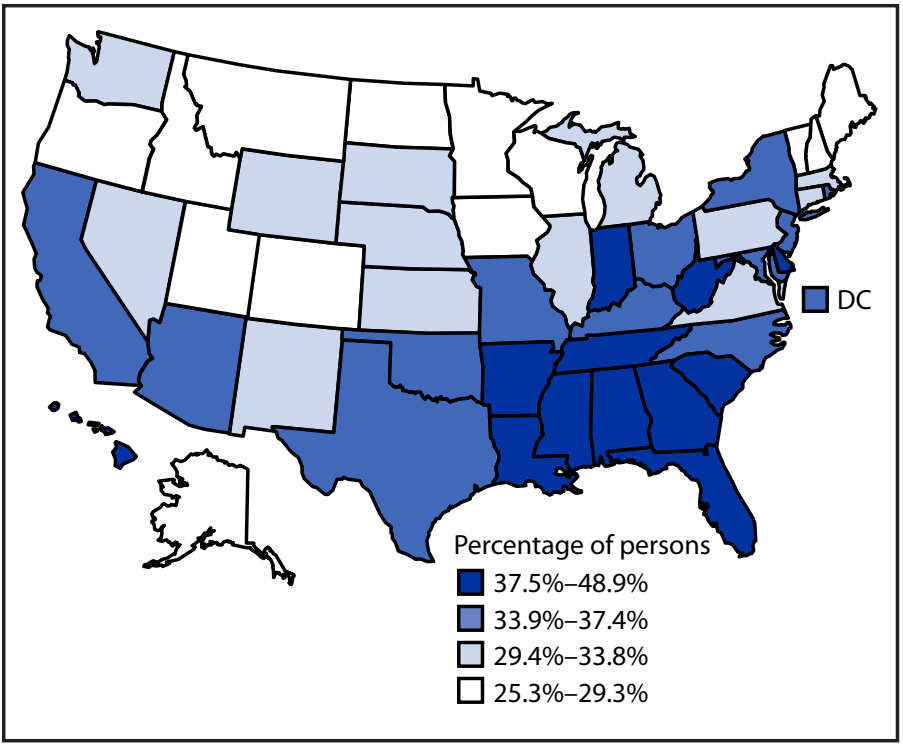

Abbreviation: $\mathrm{DC}=$ District of Columbia.

* Short sleep duration is defined as $<12$ hours for infants aged 4-11 months, $<11$ hours for children aged $1-2$ years, $<10$ hours for children aged $3-5$ years, $<9$ hours for children aged $6-12$ years, and $<8$ hours for adolescents aged 13-17 years.

were in the Midwest and Northeast (2). However, that report relied on self-report rather than parent report, did not include younger children, and excluded 20 states.

The findings in this report are subject to at least five limitations. First, sleep duration was obtained by parent report without objective measures, such as actigraphy**** or polysomnography. Second, parent reports of sleep duration might be less reliable than are self-report for older children or adolescents (3). Third, responses might be affected by recall bias, interpretation of items, or social desirability. Fourth, the statistical weighting might not completely account for nonresponse bias. Finally, the analyses with race/ethnicity were univariate and did not adjust for other covarying sociodemographic characteristics.

Insufficient sleep is an important risk factor for poor physical and mental health in infants, children, and adolescents (1). Parents can help persons aged 4 months -17 years get the sleep they need by supporting good sleep habits. Establishing a regular bedtime is a good foundation and is associated with more sleep $(9,10)$. The AASM's Bedtime Calculator ${ }^{\dagger \dagger \dagger \dagger}$ identifies appropriate bedtimes based on age-specific sleep duration recommendations and provides tips on bedtime routines

\footnotetext{
**** https://sleepeducation.org/patients/actigraphy/

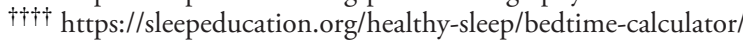

\section{Summary}

What is already known about this topic?

Infants, children, and adolescents who do not get sufficient sleep are at increased risk for adverse health outcomes. Most adolescents report sleeping less than the recommended amount. Little is known about sleep duration in infants and children.

What is added by this report?

During 2016-2018, approximately one third of children aged 4 months-17 years slept less than recommended for their age, particularly those from racial and ethnic minority groups, of low socioeconomic status, and with special health care needs. Infants, children, and adolescents with regular bedtimes were more likely to get enough sleep.

What are the implications for public health practice?

Public health practitioners, educators, and clinicians can advise parents about the importance of infants, children, and adolescents meeting recommended sleep durations, investigate the social and environmental context that affects sleep, and support parents in implementing consistent bedtimes.

for parents of infants and children and for adolescents and adults. Clinicians and educators can guide parents about the importance of sleep at all ages and discuss sleep routines and sleep problems with parents, children, and adolescents, paying attention to those with special health care needs (7). When advising parents on how to improve their infant's, child's, or adolescent's sleep, challenges that they might face because of their social and environmental context should be considered. School districts can support adequate sleep for adolescents by delaying school start times as recommended by several medical associations (2).

Corresponding author: Anne G. Wheaton, awheaton@cdc.gov, 770-488-5362.

${ }^{1}$ Division of Population Health, National Center for Chronic Disease Prevention and Health Promotion, CDC; ${ }^{2}$ Division of Human Development and Disabilities, National Center on Birth Defects and Developmental Disabilities, CDC.

All authors have completed and submitted the International Committee of Medical Journal Editors form for disclosure of potential conflicts of interest. No potential conflicts of interest were disclosed.

\section{References}

1. Paruthi S, Brooks LJ, D'Ambrosio C, et al. Consensus statement of the American Academy of Sleep Medicine on the recommended amount of sleep for healthy children: methodology and discussion. J Clin Sleep Med 2016;12:1549-61. PMID:27707447 https://doi.org/10.5664/ jcsm.6288

2. Wheaton AG, Jones SE, Cooper AC, Croft JB. Short sleep duration among middle school and high school students-United States, 2015. MMWR Morb Mortal Wkly Rep 2018;67:85-90. PMID:29370154 https://doi.org/10.15585/mmwr.mm6703a1 
3. Meltzer LJ, Brimeyer C, Russell K, et al. The children's report of sleep patterns: validity and reliability of the sleep hygiene index and Sleep Disturbance Scale in adolescents. Sleep Med 2014;15:1500-7. PMID:25441749 https://doi.org/10.1016/j.sleep.2014.08.010

4. Combs D, Goodwin JL, Quan SF, et al. Mother knows best? Comparing child-report and parent-report of sleep parameters with polysomnography. J Clin Sleep Med 2019;15:111-7. PMID:30621839 https://doi. org/10.5664/jcsm.7582

5. Hawkins SS, Takeuchi DT. Social determinants of inadequate sleep in US children and adolescents. Public Health 2016;138:119-26. PMID:27207726 https://doi.org/10.1016/j.puhe.2016.03.036

6. Grandner MA, Williams NJ, Knutson KL, Roberts D, Jean-Louis G. Sleep disparity, race/ethnicity, and socioeconomic position. Sleep Med 2016;18:7-18. PMID:26431755 https://doi.org/10.1016/j. sleep.2015.01.020

7. Rigney G, Ali NS, Corkum PV, et al. A systematic review to explore the feasibility of a behavioural sleep intervention for insomnia in children with neurodevelopmental disorders: a transdiagnostic approach. Sleep Med Rev 2018;41:244-54. PMID:29764710 https://doi.org/10.1016/j. smrv.2018.03.008
8. Liu Y, Wheaton AG, Chapman DP, Cunningham TJ, Lu H, Croft JB. Prevalence of healthy sleep duration among adults_-United States, 2014. MMWR Morb Mortal Wkly Rep 2016;65:137-41. PMID:26890214 https://doi.org/10.15585/mmwr.mm6506a1

9. Pyper E, Harrington D, Manson H. Do parents' support behaviours predict whether or not their children get sufficient sleep? A cross-sectional study. BMC Public Health 2017;17:432. PMID:28535759 https:/doi. org/10.1186/s12889-017-4334-4

10. Short MA, Gradisar M, Lack LC, et al. A cross-cultural comparison of sleep duration between US and Australian adolescents: the effect of school start time, parent-set bedtimes, and extracurricular load. Health Educ Behav 2013;40:323-30. PMID:22984209 https://doi. org/10.1177/1090198112451266 